\title{
Multilayer diamond-like amorphous carbon coatings produced by ion irradiation of polymer films
}

Sascha Buchegger ${ }^{1}$, Natascha Schuster ${ }^{1}$, Bernd Stritzker $^{1}$, Achim Wixforth ${ }^{1,2,3}$ and Christoph Westerhausen ${ }^{1,2,3}$

${ }^{1}$ Chair for Experimental Physics 1, University of Augsburg, Augsburg 86159, Germany

2 Center for NanoScience (CeNS), Ludwig-Maximilians-Universität Munich, 80799 Munich, Germany

3 Augsburg Center for Innovative Technologies (ACIT), Augsburg 86159, Germany

* Correspondence: christoph.westerhausen@gmail.com; Tel.: +49-821-598-3311

Abstract - Diamond-like carbon (DLC) is known to be a versatile coating material, being found in a wide variety of surface engineering applications. In this study, we produced DLC coatings by ion irradiation and thus reconstruction of a thin polymer film. This procedure leads to a thin diamond-like carbon layer which is characterized by its high hardness. Due to the finite ion range during implantation, however, the maximum thickness of such coatings is limited. Nevertheless, several applications for DLC coatings demand a larger thickness. We thus increased the thickness of such DLC coatings by repeatedly applying the deposition and transformation process, thus creating thicker multilayers. We show that their physical and mechanical properties like roughness, nano hardness, $\mathrm{sp}^{3}$ fraction as well as the very important adhesion of thick DLC films produced this way are very well suited for the desired applications. Although we find a small decrease in hardness and a little bit less $\mathrm{sp}^{3}$ fraction with increasing coating thickness, our results exhibit a typical hardness as well as $\mathrm{sp}^{3}$ fraction for a-C:H even for the largest coating thicknesses produced so far. 


\section{Introduction}

Depending on the actual deposition technique, diamond-like carbon (DLC) coatings represent a group of materials with a wide range of properties. For such coatings, the range of applications appears also to be very broad, ranging from protective coatings in automotive industry [1] to biomedical applications [2]. So far, DLC is either produced and deposited by an ion bombardment in plasma assisted chemical vapor deposition (PECVD) or by physical vapor deposition techniques [3,4]. Due to the excellent tribological properties as well as the corrosion resistance and good biocompatibility, DLC offers a wide-spread field of applications. It is often used in tribological systems like engine parts, in medical applications like orthopedic implants [2,5] or in protective coatings for metals to prevent the substrates from corrosion [6,7]. Since the adhesion of DLC coatings to metal substrates due to the high compressive stress of the DLC it often is not sufficiently high, a common approach is the application of adhesion promoting interlayers. For this purpose, carbide forming metals like tungsten are often used [8]. Furthermore, silicon or chromium are used in adhesion interlayers $[9,10]$. Employing dopants, it is also possible to adjust the mechanical properties of DLC. Thus, multilayer systems of DLC with a varying amount of dopants in each layer are used to reduce the internal stress and thus improve the adhesion [11]. Also, Tungsten-Carbide-DLC multilayer coatings are used to improve the adhesion [12].

Very much like the field of applications itself, also the methods of DLC production are variegated, mainly being classified into vacuum based PVD and CVD techniques. The first class mostly describes methods for the production of hydrogen free a-C and ta-C by evaporating a solid carbon target. Pulsed laser deposition, vacuum arc evaporation or magnetron sputtering are prominent examples of PVD techniques. In contrast to PVD, CVD is applicable to produce hydrogen containing DLC coatings, based on the deposition of carbon species like methane or acetylene. 
In our previous studies, a method was developed to produce DLC coatings by irradiating a polymer film with ions,[13]. Here, a polymer film was deposited by a sol-gel process onto the substrate and was subsequently transformed into DLC by ion irradiation in a plasma immersion ion implantation (PIII) process. The underlying transformation mechanism from polymer to diamond-like hydrogenated carbon is driven by the energy delivered by the impinging ions. Electronic and nuclear stopping leads to ionization, hydrogen loss and cross-linking along the trajectories of the ions as well of the carbon recoils, which in turn lead to a densification at the end of the ion trajectories. The hybridization in which the relaxing recoil atoms are binding is dependent on the local density of the material, which implicates that the transformation process has to be stopped after reaching the optimal fluence or density, respectively $[13,14]$. This technique also offers the possibility to transform a metal nanoparticle-doped polymer solution into nanoparticle-containing DLC, resulting in antimicrobial effective DLC surfaces $[15,16]$. Due to the limited ion range in solids, however, the thickness of such coatings is limited. Since there are numerous applications of coatings which require a much higher thicknesses, the extension of the coating thickness by repeatedly depositing a polymer layer and transforming it to DLC was investigated in this study. Apart from an optimization of the deposition process, here we focus on the investigation of the physical and mechanical characteristics like roughness, hardness, $\mathrm{sp}^{3}$ fraction and adhesion properties of undoped multilayer DLC coatings.

\section{Materials and methods}

A stock solution of Polyvinylpyrrolidone with a concentration of $35 \mathrm{mg} / \mathrm{ml}\left(\left[\mathrm{C}_{6} \mathrm{H}_{9} \mathrm{NO}\right]_{\mathrm{n}}, \mathrm{PVP}\right.$, M.W. 58000, ABCR GmbH) was produced by dissolving the polymer powder in Ethanol. Substrates were ultrasonically cleaned in acetone and ethanol for 20 minutes and a polymer coating was deposited by dip-coating, resulting in a homogeneous polymer film, as described before [16]. Here, the film thickness was controlled by the viscosity of the polymer solution and by the retraction speed. Silicon substrates were coated with a $126 \mathrm{~nm}$ thick PVP film. 
Subsequently, the polymer film was transformed into a diamond-like carbon coating by irradiation with high energy ions. During the ion implantation, the polymer film is continuously densified and cross-linked, eventually resulting in a 40-50 nm thick DLC layer. Ion irradiation was performed in a Plasma Immersion Ion Implantation (PIII) setup equipped with an ECR plasma source $(2.45 \mathrm{GHz})$ and a high voltage pulse generator (GBS Elektronik, RUP3). Mass flows of processing gases were controlled with separate mass flow controllers to $47.7 \% \mathrm{Ne}$, 32.7\% $\mathrm{Ar}$ and $19.6 \% \mathrm{CH}_{4}$. The working gas pressure was $3.0 \cdot 10^{-3} \mathrm{mbar}$, whereas the base pressure after evacuation was $1 \cdot 10^{-4}$ mbar. A microwave power of $800 \mathrm{~W}$ was applied. Ion Implantation was carried out by pulse biasing the substrate holder by $-20 \mathrm{kV}$ for a time of $5 \mu \mathrm{s}$ and a repetition rate of $200 \mathrm{~Hz}$. The process time was determined to be $73 \mathrm{~min}$, yielding in an implanted fluence of roughly $1 \cdot 10^{17}$ atoms $/ \mathrm{cm}^{2}$. These parameters were found and optimized in preliminary studies to result in an optimum of the physical and mechanical properties of DLC [17].

As the implantation voltage in the PIII process is limited, and it has also been found that too high pulse voltages lead to a decreasing hardness and a decreasing $\mathrm{sp}^{3}$ fraction [18-20], the thickness of one single DLC film is limited to roughly $50 \mathrm{~nm}$. Therefore, we produced thicker

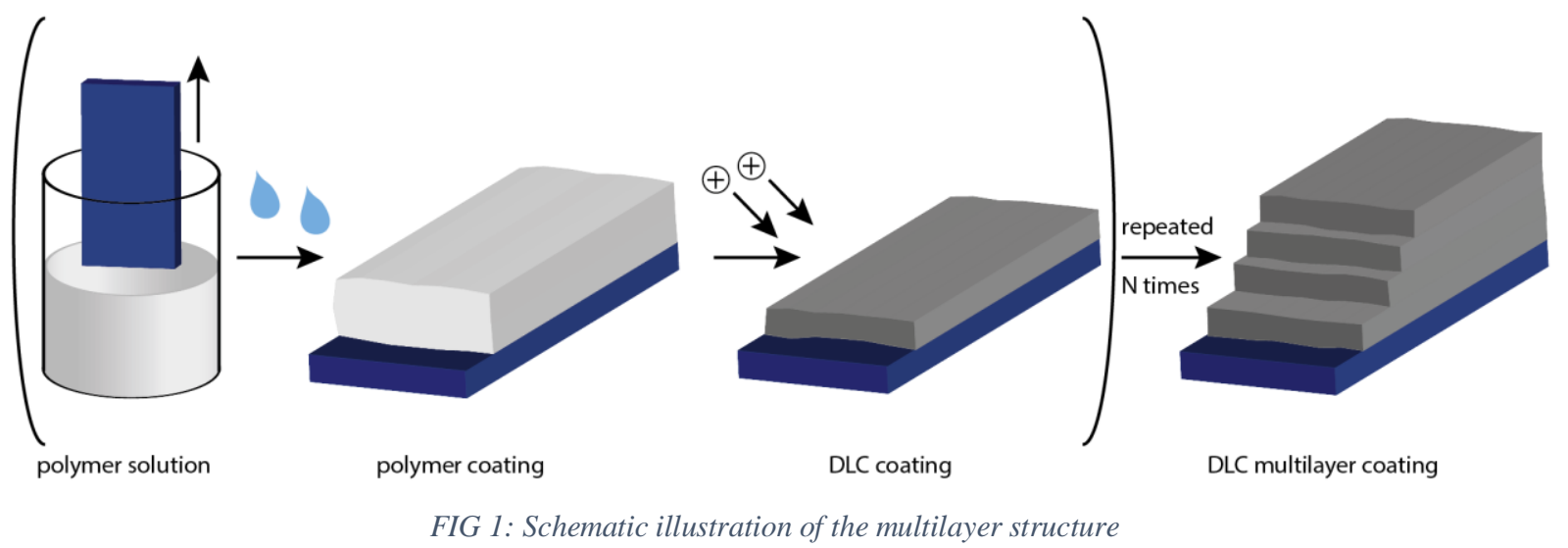

DLC films by repeatedly applying the above coating procedure (as illustrated in FIG1). Unavoidably in this first and simple approach, and due to the dip-coating-steps, the samples were exposed to air for a few minutes prior to each layer fabrication step. 
To determine the hardness of the coating, nano hardness measurements were performed, using an ASMEC UNAT nano indenter in QCSM mode. Using a Berkovich type diamond tip, a maximum force of $1 \mathrm{mN}$ was applied. For investigation of the hybridization type of the DLC layer, Raman light scattering measurements were performed. Those were carried out by exciting the sample by the $514 \mathrm{~nm}$ line of an argon-ion laser at $1 \mathrm{~mW}$. The spot was focused by a 100x microscope objective and the spectra were recorded using a CCD spectrometer. The adhesion properties were measured by nano scratch tests, using the same nano indenter as used for the hardness measurements but employing a spherical diamond tip with a diameter of 10 $\mu \mathrm{m}$. We scratched over a length of $100 \mu \mathrm{m}$ with a scratch speed of $3 \mu \mathrm{m} / \mathrm{s}$. While moving the tip, we applied a force ramp from 1 to $700 \mathrm{mN}$. To investigate the layer structure, we analyzed TEM cross section bright field images, performed with JEOL JEM 2100F. With this device, we also performed EDX line scans (200 kV, magnitude 600.000) across the Silicon-CarbonInterface. 


\section{Results}

In the contact areas of interacting tribological systems, the roughness of the friction partners is of outstanding importance. We measured the average roughness with an AFM (JPK Instruments Nano Wizard II) in contact mode. An area of $1 \mu \mathrm{m} \times 1 \mu \mathrm{m}$ was measured and the average roughness $\mathrm{R}_{\mathrm{A}}$ of this area was calculated for a range between 1 and 15 repetitions of the coating process and therefore for coating thickness between $40 \mathrm{~nm}$ to $600 \mathrm{~nm}$. As can be seen in FIG2, there occurs only a slight change in the average roughness from $R_{A}=0.34 \mathrm{~nm}$ to $R_{A}=0.39 n m$ by increasing the number of layers from one to fifteen, respectively.
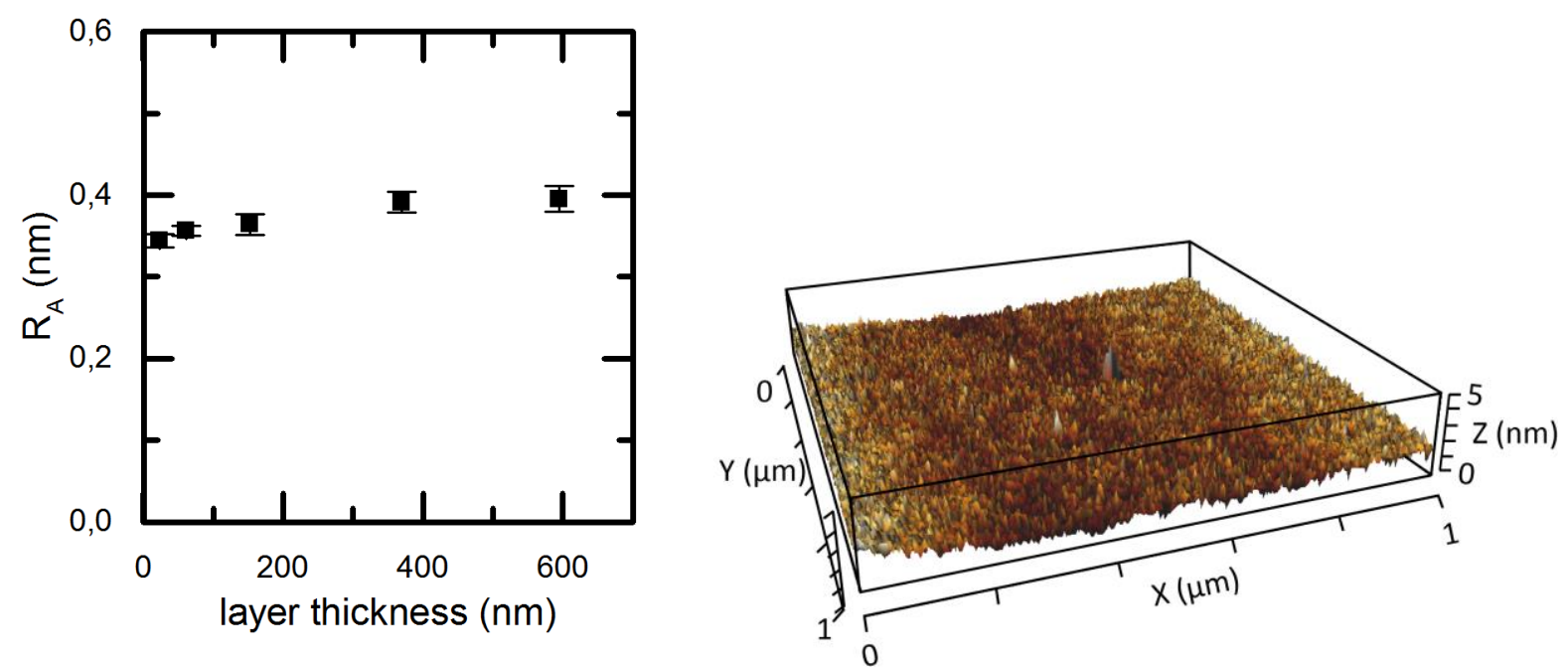

FIG 2: a) Surface roughness as function of the layer thickness b) Topology of a multilayer DLC coating (15 layers, $600 \mathrm{~nm}$ )

This change in roughness is negligible small and thus, despite the multilayer processing, the surface remains almost perfectly smooth, even for the thickest coating of $600 \mathrm{~nm}$. Fabrication and analysis of DLC multilayers on other substrates than Si-wafers show that the roughness of the substrate has a much higher impact on the surface roughness than increasing the thickness of the DLC coating. Thus, it seems to be possible to arbitrarily increase the number of layers without changing the surface roughness appreciably, which is essential for most tribological applications. Furthermore, the hardness of such materials determines the resistance to wear. 
Hence, nano hardness measurements were performed. Results of the hardness measurement as function of the layer thickness are presented in FIG3.

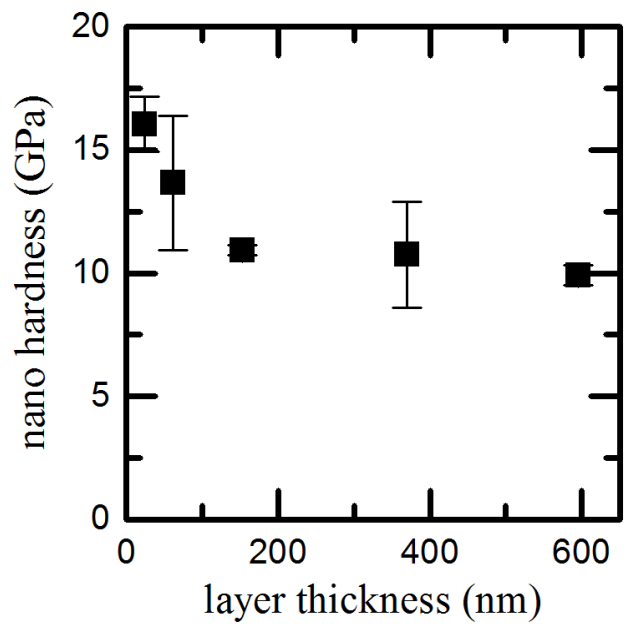

FIG 3: Nano hardness in dependence of layer thickness

By increasing the coating thickness, the hardness is decreasing from roughly $16 \mathrm{GPa}$ for a single layer to a saturation value of $10 \mathrm{GPa}$ for multiple DLC layers. According to Robertson [4], these hardness values are typical for a-C:H. For a better understanding of this hardness decrease, Raman spectroscopy measurements were made to investigate the ratio of $\mathrm{sp}^{3} / \mathrm{sp}^{2}$ hybridization types. The so-called D- and G-Peaks of the spectra were fitted by a Lorentzian and their intensity ratio $\mathrm{I}(\mathrm{D}) / \mathrm{I}(\mathrm{G})$ was calculated (see FIG4). 

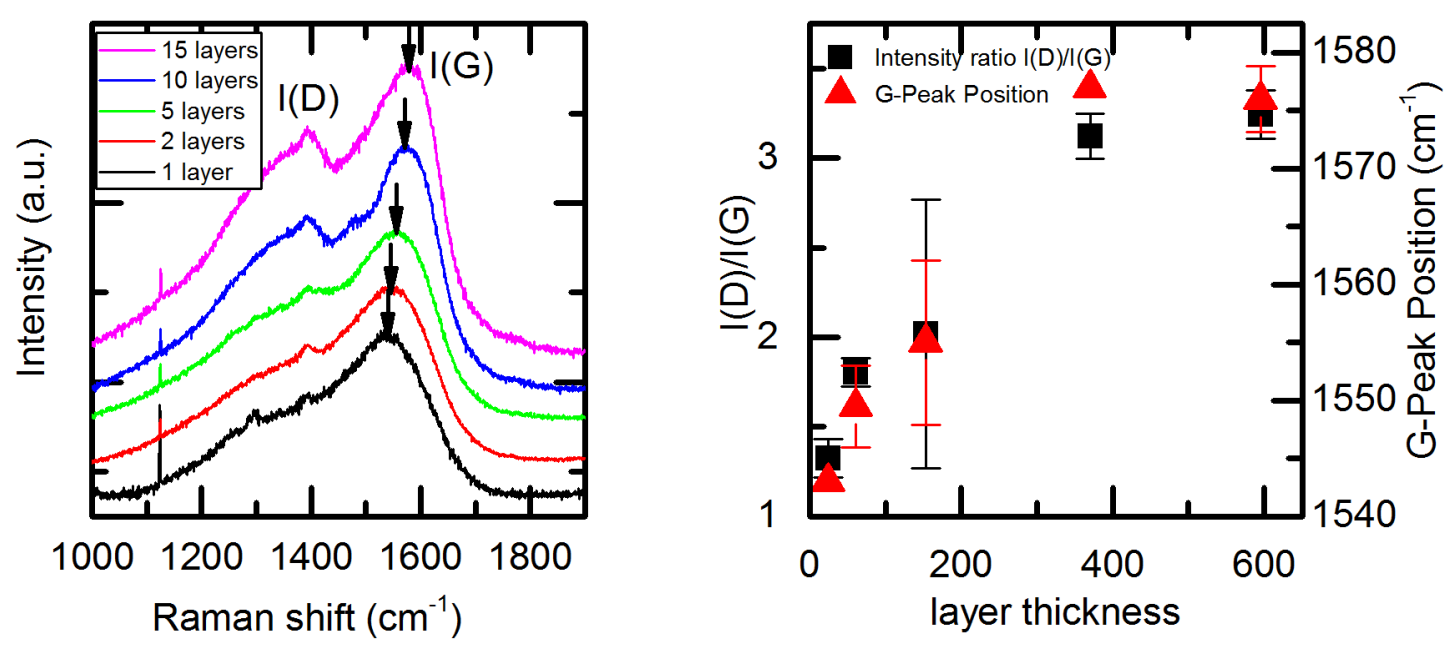

FIG 4: a) Raman spectra of DLC multilayers. The arrows indicate the maximum, identified as the G-Peak. b) Intensity ratio $I(D) / I(G)$ and $G$-Peak position as function of multilayer thickness.

Recorded spectra are presented in FIG4a and the determined intensity ratios $\mathrm{I}(\mathrm{D}) / \mathrm{I}(\mathrm{G})$ as well as the position of the G-Peak are shown in FIG4b. As can be seen in FIG4b, the intensity ratio $\mathrm{I}(\mathrm{D}) / \mathrm{I}(\mathrm{G})$ is increasing with increasing number of DLC layers. Furthermore, the position of the G-peak in the spectra shows exactly the same trend as the intensity ratio. Both measured values are converging against a saturation value. The G-Peak position shifts from $1543 \mathrm{~cm}^{-1}$ for one layer to higher wave numbers and converges to $1575 \mathrm{~cm}^{-1}$ for a large number of layers (15 or more). The $\mathrm{I}(\mathrm{D}) / \mathrm{I}(\mathrm{G})$ ratio was 1.3 for one DLC layer whilst it shows a saturation value of roughly 3.2 for 15 DLC layers or more. By comparison of these values with literature values $[4,21]$, one can estimate the fraction of $\mathrm{sp}^{3}$ type hybridizations in this coatings.

For one single DLC layer, we estimate a percentage of roughly $36 \% \mathrm{sp}^{3}$ type hybridizations, whereas this fraction declines to $25 \%$ in the case of the thickest coating analyzed (15 DLC layers). We ascribe this drop in $\mathrm{sp}^{3}$ fraction to the penetration range of the impinging ions being larger than the thickness of the topmost DLC layer in each transformation step. Due to this fact, the subjacent DLC layer formed by the preliminary coating process was penetrated by the ions of the following ion implantation process and therefore the overall implantation fluence of the subjacent layer is higher than the ideal fluence. Higher implantation doses than the ideal dose predominantly lead to the formation of $\mathrm{sp}^{2}$ hybridizations and therefore to a decrease in $\mathrm{sp}^{3}$ 
fraction, a process being called graphitization [18-20]. This hypothesis for the decreasing diamond-like character of the analyzed samples is strongly supported by additional sets of measurements, where the process time and thus the implanted fluence was increased. By doubling the implantation fluence from $1 \cdot 10^{17}$ atoms $/ \mathrm{cm}^{2}$ to $2 \cdot 10^{17}$ atoms $/ \mathrm{cm}^{2}$, the estimated $\mathrm{sp}^{3}$ fraction dropped from roughly $39 \%$ to $31 \%$. As the hardness is determined by the $\mathrm{sp}^{3}$ fraction and nano hardness as well as the $\mathrm{sp}^{3}$ fraction showed the same decreasing trend, both trends can be explained by a graphitization of the DLC layer. Moreover, temperature effects as reported earlier [22] can be excluded, as the temperature during the PIII process is significantly lower. Nevertheless, all spectra measured are still indicating an amount of $\mathrm{sp}^{3}$ hybridized carbon atoms typical for a-C:H [4]. Despite this we do not recommend to lower the fluence, as it is of great importance to crosslink the DLC coating to the substrate and therefore let the ion range exceed the final DLC film thickness.

Since the film adhesion is of great importance, we carried out nano scratch tests to investigate the adhesion properties of this coating. The result of a typical scratch test on a $600 \mathrm{~nm}$ DLC multilayer coating is illustrated in FIG5.

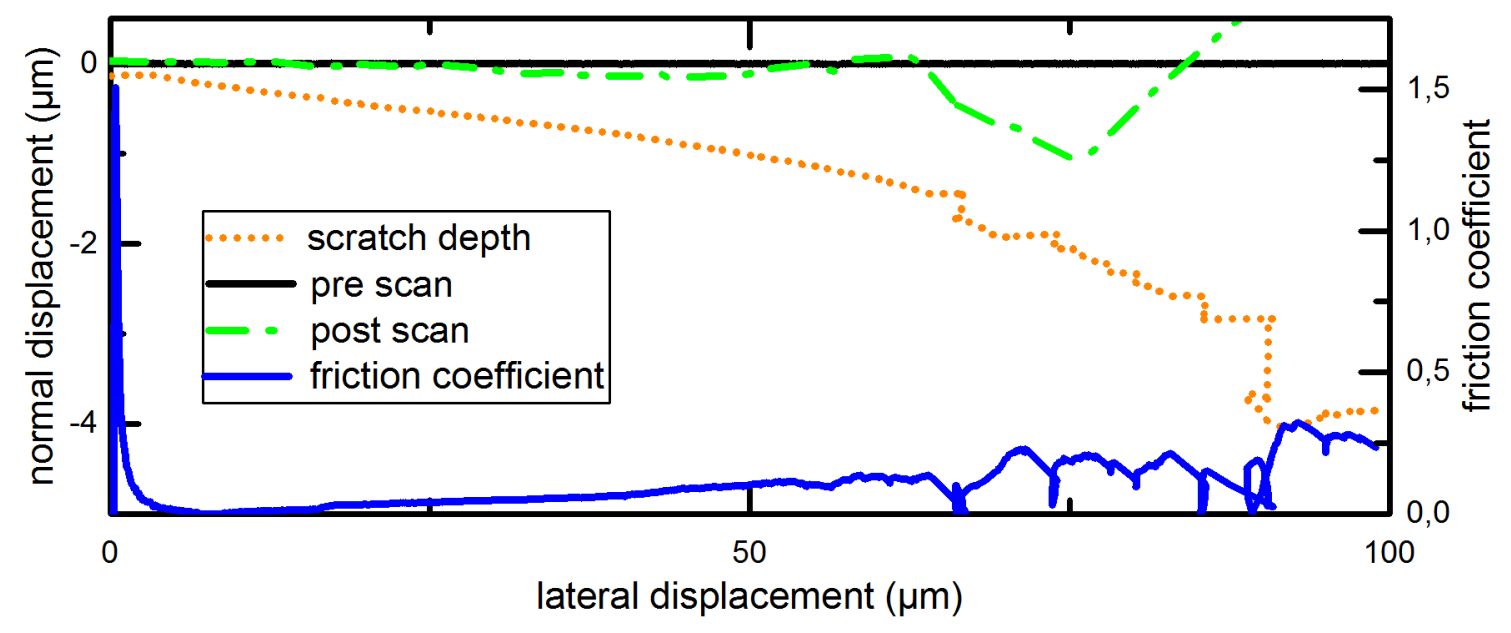

FIG 5: Typical results of a scratch test of a DLC coating

The critical force $\mathrm{F}_{\mathrm{C}}$ at which the coating is fully delaminating remained almost constant at roughly $350 \pm 50 \mathrm{mN}$ for all samples measured. This value exceeds that one for DLC samples produced by PECVD technique [10], even though we did not use a Cr-Interlayer as an adhesion 
promoter, like it was used by Wei et al. [10]. Wei reported a critical load of $265 \mathrm{mN}$ for a film thickness of $200 \mathrm{~nm}$, which decreased with higher film thickness. For a $600 \mathrm{~nm}$ thick film, the critical load was reduced to $95 \mathrm{mN}$. In contrast, the samples produced with our method were independent of the number of DLC layers respectively the coating thickness. We attribute this to the low internal stress of the DLC coating which is not significantly increased by adding additional DLC layers. Consequently, the adhesion properties are not affected by the thickness of the coating. No significant stress induced bending was detected either by performing profilometer measurements over a distance of $2000 \mu \mathrm{m}$. Assuming the height uncertainty to be lower than $2 \mathrm{~nm}$ and using Stoney's equation [23], we estimated the internal stress to be less than 0,04 GPa, being very small in contrast to DLC coatings fabricated in PECVD and FVCA, which exhibit an internal stress of 1 to $8 \mathrm{GPa}$ [24-26].

Additionally to the scratch tests, we performed adhesion tests according to the German standard VDI3198 [27]. Based on the Rockwell C indentation test, a conical tip was forced to penetrate the surface and the shape of the imprint was optically investigated. The imprint of the indentation test is an indicator for the adhesion properties of the coating to the substrate. According to VDI3198, the imprints are optically classified in six categories, starting with 'very good adhesion, (HF1)' when only micro cracks are visible around the imprint to 'poor adhesion, (HF6)' where extensive delamination occurs in the vicinity of the imprint. We tested coatings
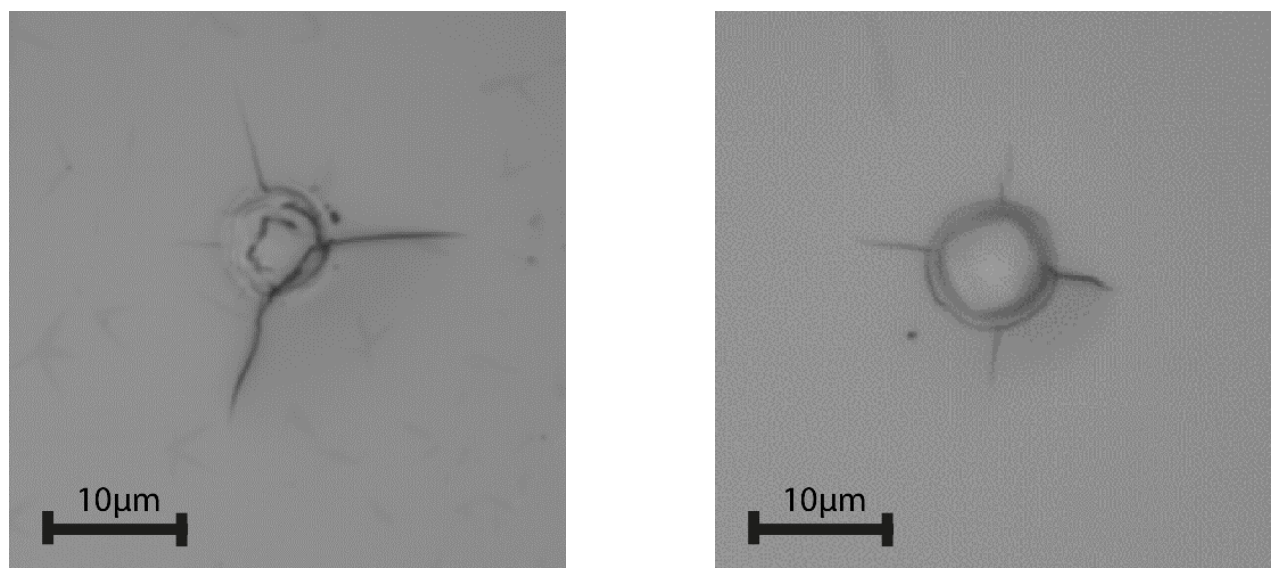

FIG 6: Imprints of the adhesion test according to VDI 3198 of one single DLC layer (left) and 15 layers of DLC (right). 
of single DLC layers and multilayer coatings consisting of 15 layers of DLC. Three imprints for each sample were investigated. Typical results are shown in FIG 6. As only micro cracks were visible, both samples were classified in HF1, indicating strong adhesion. Due to this classification and to the results of the scratch test, we conclude that the adhesion of multilayer DLC coatings produced with this technique is independent of the number of layers.

Again, the very good adhesion of one single layer is not deteriorating by repeatedly coating the sample. In contrast, thick DLC coatings produced with standard CVD-techniques show a thickness-dependent adhesion, becoming worse for thicker films. Another big advantage of our method in comparison to other DLC-coating techniques is that no adhesion-promoting interlayer is necessary. Due to ion-induced cross-linking with the substrate and reduced internal stress of the DLC coating, adhesion to the substrate is even better than the ones involving an interlayer [10]. In analogy to doped multilayer coatings [11], for our coatings it is thus possible to actually customize and tailor the hardness and $\mathrm{sp}^{3}$ fraction by merely adjusting the implantation-fluence. 
To study the internal structure of the multilayer coatings in more detail, we prepared TEM cross section images of the DLC coating consisting of 15 layers (see FIG 7). Apart from the very last single layer, all interfaces between the layers can be clearly seen. The change in brightness in the vicinity of the interfaces between two DLC layers might be understood by a selective sputter rate at the edges during the preparation process of the TEM cross section sample and consequential fluctuations in thickness. The differing sputter rate is most likely caused by a difference in the atomic structure and accordingly bonding forces. An EDX mapping of the sample did not show any contamination with other elements along these interfaces. This

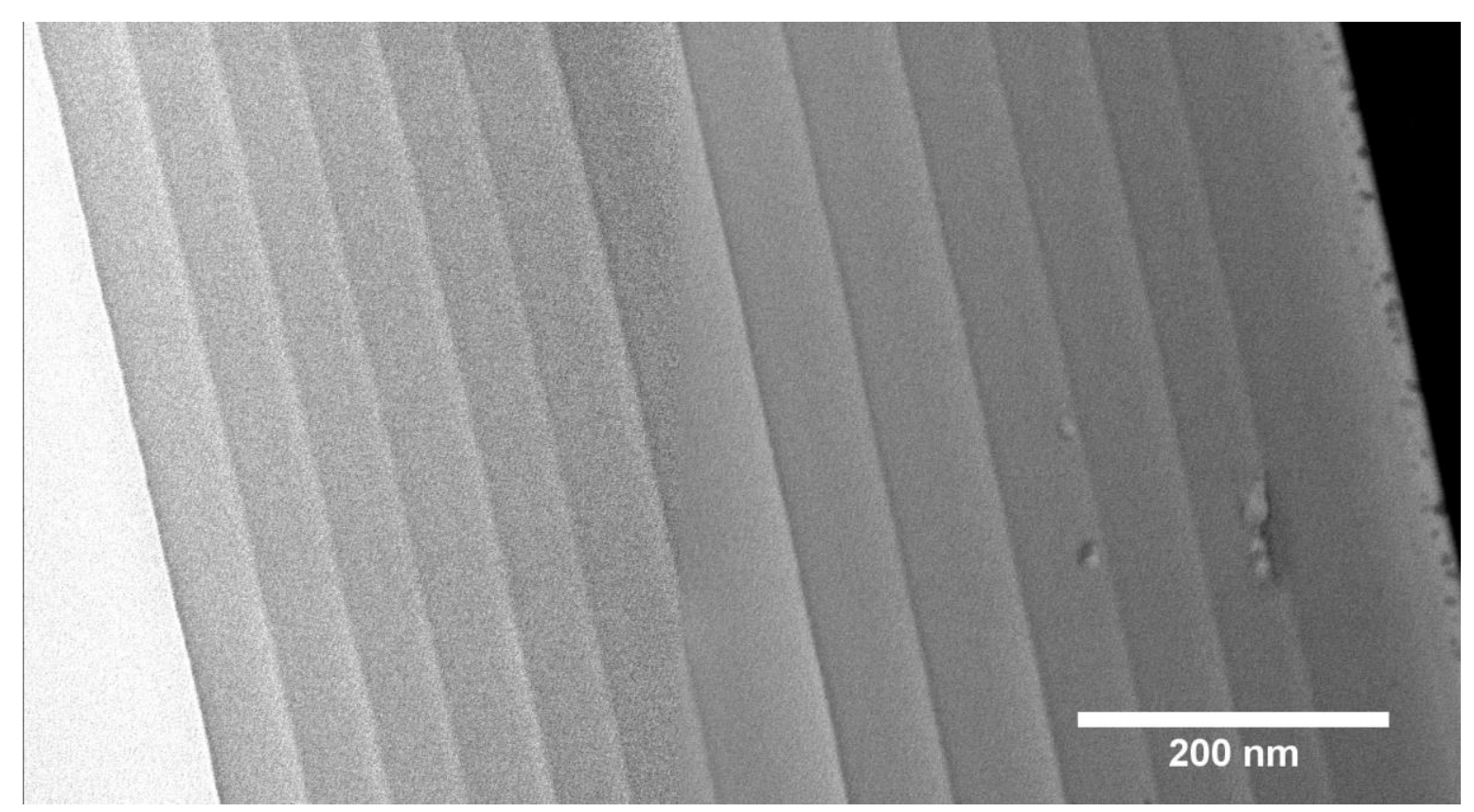

FIG 7: TEM cross section image of 15 layer of DLC coating, composed of two locally overlapping high resolution bright field images. 

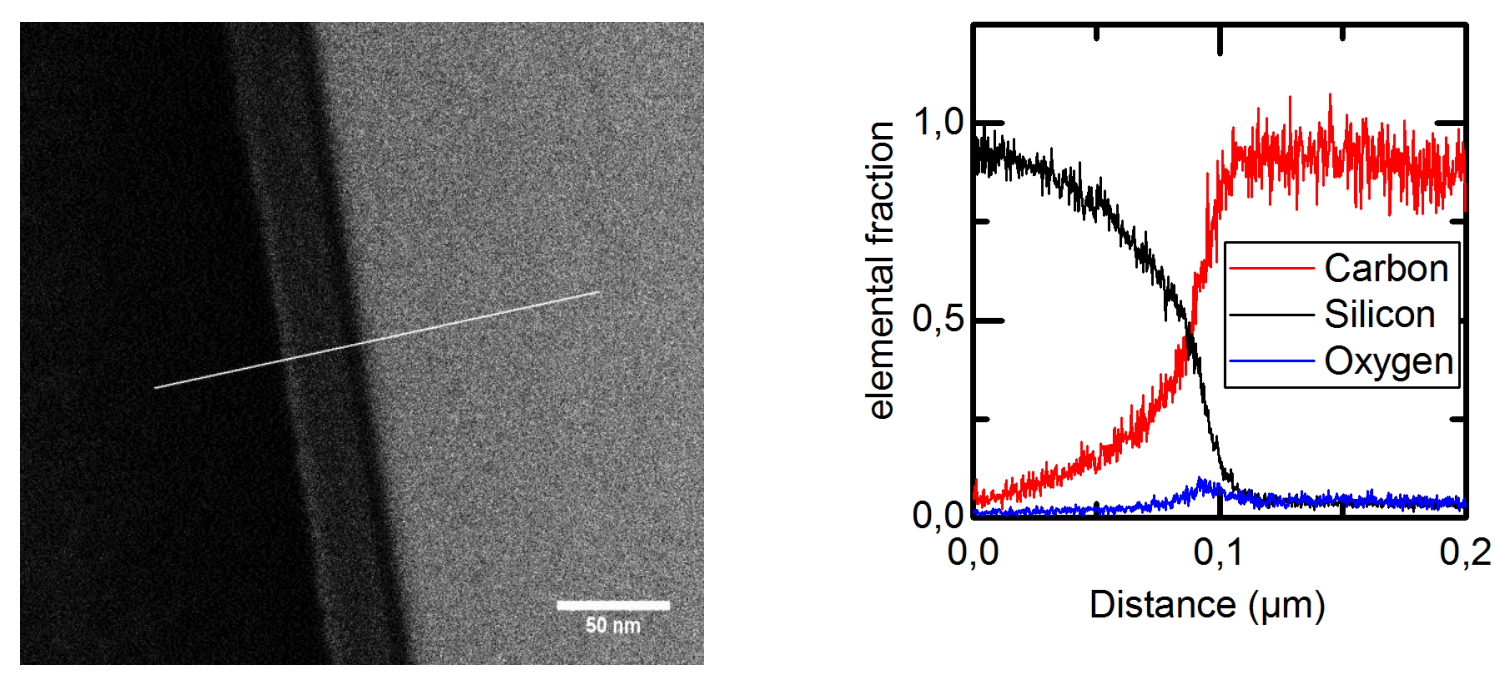

FIG 8: EDX line scan across the silicon-DLC interface show a gradient in carbon fraction within the silicon substrate.

behavior may be an explanation for the small internal stress, which does not increase with increasing coating thickness. An EDX line scan across the silicon-DLC interface (see FIG 8) exhibits a gradient of the carbon fraction under the silicon surface, being caused by carbon recoil atoms during the implantation process. The oxygen signal is assigned to the silicon oxide layer at the surface of the silicon wafer and therefore marks the surface of the silicon wafer. The elemental mixing within the substrate explains the very good adhesion of the DLC coating. Additional ERDA and RBS measurements were performed to clarify the hydrogen content as well as the density of the coating using the coating thickness and the areal density. The hydrogen content of our carbon structure is about 34 at\%, which is typical for a-C:H [4]. Moreover, a typical value of $1.7 \mathrm{~g} / \mathrm{cm}^{3}$ was measured for the a-C:H.

\section{Conclusion}

We successfully deposited a variety of DLC multilayer films onto silicon substrates. Roughness, hardness and bonding structure as well as coating adhesion were analyzed. Our measurements indicated very smooth and well-adherent DLC coatings. Very importantly, these two quality measures remained almost constant for an increasing number of layers. By measuring the nano hardness, however, we observed a decrease in hardness with increasing 
number of DLC layers. This hardness decrease is determined by the fraction of $\mathrm{sp}^{3}$ hybridizations within the carbon network which shows the same trend as the hardness for an increasing number of layers. This decrease in hardness and $\mathrm{sp}^{3}$ fraction results from an overlap in the ion penetration ranges during the implantation process which in turn results in an effective fluence for each subjacent layer, being larger than the optimal fluence for polymer-to -DLC transformation. This leads to a decrease in diamond-like character of the coating, and to graphitization. Nevertheless, all measured data showed typical values for a-C:H, even for the thickest coating. A possibility to avoid this graphitization is to adjust the fluence in a way where the effective fluence of each layer is very close to the optimal fluence, but still the crosslinking of the layers is guaranteed. Hence, we are able to produce thick multilayer DLC coatings with high hardnesses and good film adhesion, which can for example be used for functional multilayer systems.

\section{Disclosures}

The authors have no financial conflicts of interest.

\section{Acknowledgments}

The authors would like to thank the "Deutsche Forschungsgemeinschaft (DFG)" for the financial support of this research work under the contract "Erkenntnistransferprojekt Antibakterielle und abriebarme Beschichtung von Gleitflächen in orthopädischen Implantaten”. Moreover, the authors thank Dr. Rudolf Herrmann for fruitful discussions and Michael Weinl for technical assistance. 


\section{References}

[1] Hauert R. An overview on the tribological behavior of diamond-like carbon in technical and medical applications. Tribol Int 2004;37:991-1003. doi:10.1016/j.triboint.2004.07.017.

[2] Roy RK, Lee K-R. Biomedical applications of diamond-like carbon coatings: a review. J Biomed Mater Res B Appl Biomater 2007;83:72-84. doi:10.1002/jbm.b.30768.

[3] Grill A. Diamond-like carbon: State of the art. Diam Relat Mater 1999;8:428-34. doi:10.1016/S0925-9635(98)00262-3.

[4] Robertson J. Diamond-like amorphous carbon. Mater Sci Eng R Reports 2002;37:129281. doi:10.1016/S0927-796X(02)00005-0.

[5] Roy ME, Whiteside LA, Katerberg BJ. Diamond-like carbon coatings enhance scratch resistance of bearing surfaces for use in joint arthroplasty: hard substrates outperform soft. J Biomed Mater Res B Appl Biomater 2009;89:527-35. doi:10.1002/jbm.b.31244.

[6] Cui M, Pu J, Zhang G, Wang L, Xue Q. The corrosion behaviors of multilayer diamond-like carbon coatings: Influence of deposition periods and corrosive medium. RSC Adv 2016;6:28570-8. doi:10.1039/C6RA05527C.

[7] Mansano RD, Massi M, Mousinho AP, Zambom LS, Neto LG. Protective carbon layer for chemical corrosion of stainless steel. Diam Relat Mater 2003;12:749-52. doi:10.1016/S0925-9635(02)00270-4.

[8] Lee K-R, Yong Eun K, Kim I, Kim J. Design of W buffer layer for adhesion improvement of DLC films on tool steels. Thin Solid Films 2000;377-378:261-8. doi:10.1016/S0040-6090(00)01429-2.

[9] Cemin F, Bim LT, Menezes CM, Maia da Costa MEH, Baumvol IJR, Alvarez F, et al. The influence of different silicon adhesion interlayers on the tribological behavior of DLC thin films deposited on steel by EC-PECVD. Surf Coatings Technol 2015;283:115-21. doi:10.1016/j.surfcoat.2015.10.031. 
[10] Wei C, Yen J-Y. Effect of film thickness and interlayer on the adhesion strength of diamond like carbon films on different substrates. Diam Relat Mater 2007;16:1325-30. doi:10.1016/j.diamond.2007.02.003.

[11] Delplancke-Ogletree MP, Monteiro OR. Wear behavior of diamond-like carbon/metal carbide multilayers. Surf Coatings Technol 1998;108-109:484-8. doi:10.1016/S02578972(98)00572-6.

[12] Rincón C, Zambrano G, Carvajal A, Prieto P, Galindo H, Martlínez E, et al. Tungsten carbide/diamond-like carbon multilayer coatings on steel for tribological applications. Surf Coatings Technol 2001;148:277-83. doi:10.1016/S0257-8972(01)01360-3.

[13] Schwarz F, Stritzker B. Plasma immersion ion implantation of polymers and silverpolymer nano composites. Surf Coatings Technol 2010;204:1875-9. doi:10.1016/j.surfcoat.2009.10.044.

[14] Schwarz FP. Ioneninduzierte Umwandlung von Polymerschichten zu diamantähnlichem Kohlenstoff mit darin enthaltenen Silber-Nanopartikeln. Dissertation, Augsburg, 2010.

[15] Gorzelanny C, Kmeth R, Obermeier A, Bauer AT, Halter N, Kumpel K, et al. Silver nanoparticle-enriched diamond-like carbon implant modification as a mammalian cell compatible surface with antimicrobial properties. Sci Rep 2016;6:22849. doi:10.1038/srep22849.

[16] Buchegger S, Vogel C, Herrmann R, Stritzker B, Wixforth A, Westerhausen C. Antibacterial metal ion release from diamond-like carbon modified surfaces for novel multifunctional implant materials. J Mater Res 2016;31:2571-7. doi:10.1557/jmr.2016.275.

[17] Schwarz F, Thorwarth G, Wehlus T, Stritzker B. Silver nanocluster containing diamond like carbon. Phys Status Solidi 2008;205:976-9. doi:10.1002/pssa.200778327. 
[18] Krauser J, Nix A-K, Gehrke H-G, Hofsäss H, Trautmann C, Weidinger A, et al. Ion track lithography and graphitic nanowires in diamondlike carbon. J Vac Sci $\{\&\}$ Technol B Microelectron Nanom Struct 2008;26:2468-72. doi:10.1116/1.3010738.

[19] Sorkin A, Adler J, Kalish R. Computer simulations of damage due to passage of a heavy fast ion through diamond. Phys Rev B 2004;70. doi:10.1103/PhysRevB.70.064110.

[20] Galvão JR, Luce FP, Baptista DL, da Costa MEMM, Lepienski CM, Zawislak FC. Ion irradiation effects on a-C: H, a-C:N:H and a-C:F:H films. Nucl Instruments Methods Phys Res Sect B Beam Interact with Mater Atoms 2006;249:409-13. doi:10.1016/j.nimb.2006.04.040.

[21] Ferrari AC, Robertson J. Interpretation of Raman spectra of disordered and amorphous carbon. Phys Rev B 2000;61:14095-107. doi:10.1103/PhysRevB.61.14095.

[22] Conway NMJ, Ferrari AC, Flewitt AJ, Robertson J, Milne WI, Tagliaferro A, et al. Defect and disorder reduction by annealing in hydrogenated tetrahedral amorphous carbon. Diam Relat Mater 2000;9:765-70. doi:10.1016/S0925-9635(99)00271-X.

[23] Wei Q, Narayan RJ, Sharma AK, Sankar J, Narayan J. Preparation and mechanical properties of composite diamond-like carbon thin films. J Vac Sci $\{\&\}$ Technol A Vacuum, Surfaces, Film 1999;17:3406. doi:10.1116/1.582074.

[24] Bonetti LF, Capote G, Santos L V, Corat EJ, Trava-Airoldi VJ. Adhesion studies of diamond-like carbon films deposited on Ti6Al4V substrate with a silicon interlayer. Thin Solid Films 2006;515:375-9. doi:10.1016/j.tsf.2005.12.154.

[25] Wang P, Wang X, Xu T, Liu W, Zhang J. Comparing internal stress in diamond-like carbon films with different structure. Thin Solid Films 2007;515:6899-903. doi:10.1016/j.tsf.2007.02.069.

[26] Sheeja D, Tay BK, Yu L, Lau SP. Low stress thick diamond-like carbon films prepared by filtered arc deposition for tribological applications. Surf Coatings Technol 
2002;154:289-93. doi:10.1016/S0257-8972(02)00005-1.

[27] Vidakis N, Antoniadis A, Bilalis N. The VDI 3198 indentation test evaluation of a reliable qualitative control for layered compounds. J Mater Process Technol 2003;143144:481-5. doi:10.1016/S0924-0136(03)00300-5. 